\title{
Lymphangiectasia haemorrhagica conjunctivae
}

\begin{abstract}
Purpose To re-describe a condition that has not been mentioned in the literature for more than four decades and to outline a new method of treatment of the pathology using an argon laser.

Methods Nine patients were identified over a 2 year period who fitted a clinical picture consistent with the features of lymphangiectasia haemorrhagica. Three were treated for frequent recurrent haemorrhages with a newly developed technique using the argon laser and followed for up to 2 years. Results None of the three treated patients has had a recurrence of the condition to date. In the six untreated patients the condition resolved spontaneously after repeated attacks over between 8 months and 8 years.

Conclusions Lymphangiectasia haemorrhagica conjunctivae is probably more common than expected and may resolve spontaneously, but is readily amenable to treatment with the argon laser if attacks become frequent, uncomfortable or unsightly.
\end{abstract}

Key words Argon laser, Lymphangiectasia

\section{Clinical features}

Lymphangiectasia haemorrhagica is a condition that appears to be little recognised. There has been no mention of it in the literature for more than four decades and the most recent textbook to describe the disorder was Duke Elder's Diseases of the Outer Eye published in 1965. The condition involves sudden, rapid filling of the conjunctival lymphatics with blood by retrograde filling from the conjunctival vessels. The hallmark of the condition is that the blood clears almost as rapidly as it arrives and almost always within 3-4 days.

A typical history in this series of nine patients was of a foreign body sensation and recurrent 'bleeding on the front of the eye' with no obvious precipitating factors such as surgery, trauma, clotting abnormalities, coughing or straining. The 'whole of the white of the eye became covered in blood' in one patient and typically resolved completely in
JONATHAN LOCHHEAD, LARRY BENJAMIN
2-4 days. The nature of this condition contrasts with that of recurrent subconjunctival haemorrhage by resolving very rapidly and completely over this short period. Careful examination frequently reveals the broken column of blood with segmented constrictions and balloon-shaped dilatations characteristic of this condition (Figs. 1, 2). The distended ampoules of the lymphatic vessels involved may also demonstrate a horizontal level of blood where they are only partially filled. These features are quite distinct from the diffuse haemorrhage visible following a subconjunctival haemorrhage, which may take 10-14 days to clear.

In the previous literature, treatment has been described by a surgical means, excising the pathological communication, ${ }^{1}$ perhaps with the risk of subsequent haemorrhage, or by a process of diathermic coagulation. ${ }^{2}$ The treatment used here is similar to the latter but exploits the magnification and precision of an argon laser.

\section{Treatment protocol}

Amethocaine 1\% drops were applied to the conjunctival sac. The laser was used in its Green mode at a relatively low power $(0.3 \mathrm{~W})$ and $0.1 \mathrm{~s}$ duration. Approximately 20-30 burns of $100 \mu \mathrm{m}$ in diameter were required on each occasion to obliterate the junction between the blood and lymph vessels. Uptake varied according to the amount of blood in the tissues. Very little, if any, discomfort was experienced by any of the three treated patients.

\section{Results (Table 1)}

At the time of presentation only two patients had visible signs. One of these was treated with an argon laser and to date, within a 12 month period, has had no further trouble. Another patient who had suffered three attacks within a 3 month period returned to the clinic during a recurrence and was subsequently treated, again using an argon laser. She has remained symptom free over the last 2 years.

A third treated patient returned to the clinic with 'another episode' approximately 3 weeks following treatment. Interestingly, on this

\author{
J. Lochhead \\ Department of \\ Ophthalmology \\ St Bartholomew's Hospital \\ QEII Wing \\ West Smithfield \\ London EC1A 7BE, UK \\ L. Benjamin \\ Stoke Mandeville Hospital \\ Mandeville Road \\ Aylesbury \\ Buckinghamshire HP21 8AL, \\ UK \\ Fax: +44 (0)1296315037
}




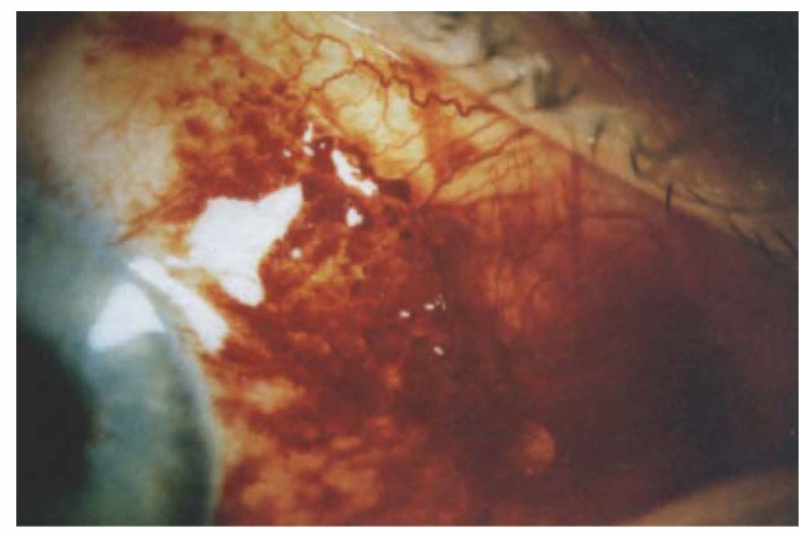

Fig. 1. A photograph of dilated lymph vessels filled with blood in the inferonasal conjunctiva. This was taken during the resolving phase of an attack.

occasion the haemorrhage was entirely subconjunctival in a region adjacent to the previous treatment. There was no visible haemorrhage into the lymphatics of the treated area or elsewhere and therefore no further treatment was considered.

The remaining patients were asked to return for review immediately on recurrence of the condition. To date none have returned and it is interesting to speculate why this might be.

\section{Pathophysiology}

The conjunctivae contain two vascular systems - a superficial and a deep plexus - between which is a free anastomosis in the loose subconjunctival connective tissue. The superficial vessels contribute primarily to the perilimbal plexus and may only be visible in congested states. The deep vessels originate from the nasal and lacrimal branches to the eyelids. They are clearly visible in the anterior segment from their origins in the fornices.

The conjunctivae are the only components of the globe and orbit to have a lymphatic drainage system. They can be divided into several groups whose channels drain directly into the superficial venous plexus. An incomplete pericorneal lymphatic ring (circulus lymphaticus) forms a rich network $1 \mathrm{~mm}$ wide of tiny lymphatic vessels surrounding the edge of the cornea. From here originate lymphatic channels that initially radiate and then become concentric with the corneal margin at about $4-5 \mathrm{~mm}$ from their origin. Large collector channels then emerge circumferentially $7-8 \mathrm{~mm}$

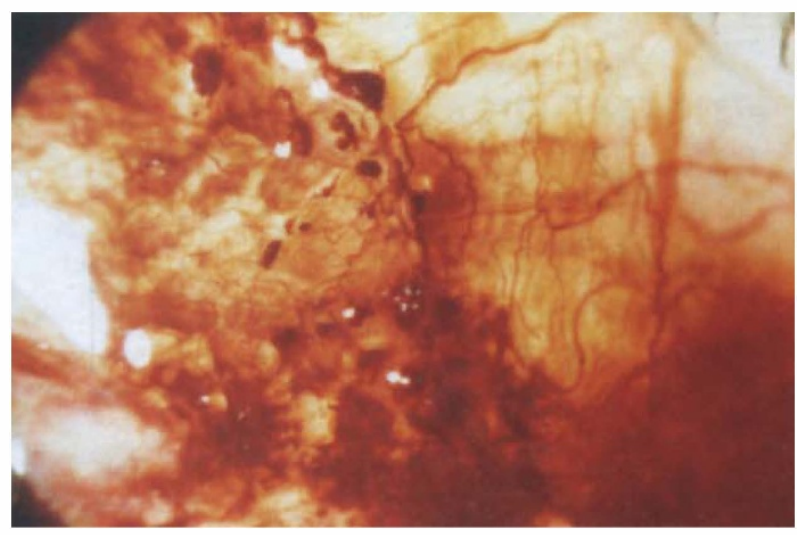

Fig. 2. A close-up of the conjunctiva seen in Fig. 1 showing the dilated lymph channels, which when not filled with blood would be transparent. Two days after this picture was taken the blood had virtually completely disappeared from the lymphatic system.

behind the limbus. These drain into the main lymph channels, which are two in number and pass from the nasal to the temporal areas of the conjunctiva. The superior channel passes around the upper half of the cornea and the inferior one around the lower half. These two channels may then fuse to form one principal trunk or may remain separate as they pass to the external angles. There are two recurrent collector channels that are smaller in size than the main collectors: a superonasal recurrent collector draining the upper nasal quadrant and a temporal pericorneal descending collector with a recurrent limb. Both drain via the medial canthus. Laterally placed lymphatic channels proceed to drain into the pre-auricular lymph nodes, while those located medially proceed to the submaxillary nodes. They appear as clear, dilated, tortuous, sausage-like lymphatic vessels (not to be confused with conjunctival lymphatic cysts) and are thought to result from a local disturbance in lymphatic flow. Sometimes these lymphangiectasia are associated with haemorrhage around them (subconjunctival haemorrhage) and occasionally with haemorrhage into them. This is then termed lymphangiectasia haemorrhagica and is thought to evolve from an abnormal connection established between a blood vessel (of the deep venous plexus) and the lymphatic vessel such that intermittent episodes of retrograde flow result in the lymphatic channel filling with blood.

Table 1. Details of the nine patients in this series.

\begin{tabular}{|c|c|c|c|c|c|}
\hline Age (years) & Sex & Location & Duration $^{a}$ & Treatment & Outcome \\
\hline 60 & $\mathrm{~F}$ & Medial & 18 months & Nil & Spontaneous resolution \\
\hline 54 & $\mathrm{M}$ & Lateral & 8 years & Nil & Spontaneous resolution \\
\hline 58 & $\mathbf{M}$ & Lateral & 6 months & Argon laser & No recurrence \\
\hline 53 & $\mathrm{M}$ & Lateral & 2 years & Nil & Spontaneous resolution \\
\hline 32 & $\mathbf{F}$ & Lateral & 12 months & Argon laser & No recurrence \\
\hline 40 & $\mathrm{M}$ & Medial & 12 months & Nil & Spontaneous resolution \\
\hline 48 & M & Lateral & 8 months & Nil & Spontaneous resolution \\
\hline 37 & $\mathbf{F}$ & Lateral & 5 months & Argon laser & No recurrence \\
\hline 52 & $\mathrm{M}$ & Medial & 10 months & Nil & Spontaneous resolution \\
\hline
\end{tabular}

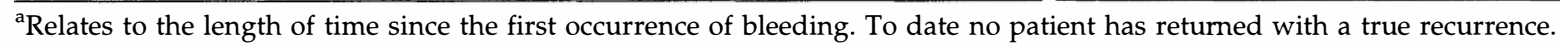




\section{Discussion}

Following the first description of lymphangiectasia haemorrhagica by Leber in 1880, there have been a number of very well documented cases such as that of Stepanik, ${ }^{3}$ where the lymphatics of a 12-year-old girl became suffused with blood each night only to clear up within hours the following day.

Histological studies were performed in the past ${ }^{1,2}$ that confirmed the nature of the condition but did not shed any light on the possible mechanisms involved. Conrads and Kuhnhardt ${ }^{1}$ found signs of chronic inflammation and went on to postulate that this could promote the formation of connective tissue strictures or septa within the lymph spaces and thus contribute to the characteristic segmented appearance. No conjunctival lymph nodes have ever been identified. ${ }^{4}$

The pattern of the lymphatics also differs in relation to their depth within the conjunctiva. There are three zones: a superficial zone lying just below the epithelium consisting of very fine lymphatics and then a slightly deeper middle zone containing the typically larger vessels; there are no lymphatics to be found in the deepest of these zones. The superficial fine network is composed of vessels that are similar in size to the medium-sized veins of the area. They communicate with the deeper network which contains vessels some 2-4 times larger than the veins of this area. It is these vessels that are primarily identified in lymphangiectasia haemorrhagica. They are very irregular in diameter with aneurysm-like outpouchings and valve-like constrictions that often give them a beaded appearance. In older individuals there is an increasing tendency for these valvules to narrow. Failure of these valvular mechanisms may be responsible for blood gaining access to the lymphatics and might explain the rapid filling of the lymph vessels with blood. Dispersal of the blood is also rapid and this may be due to direct emptying of the blood into the connecting veins from whence it came, rather than having to be removed as in the case of a subconjunctival haemorrhage.

The patients who have not, presumably, had recurrences may have closed off the connection between lymph and blood vessels by fibrosis after repeated attacks.

\section{Conclusion}

Lymphangiectasia haemorrhagica may be more common than expected and an awareness of its characteristics will be useful in examining future recurrent haemorrhages of the bulbar conjunctiva. Those patients presenting with haemorrhage that clearly has a lymphatic component and who particularly complain of associated discomfort, or voice concerns about the cosmetic appearance accompanying recurrent haemorrhages, may be considered for treatment with the argon laser. The main diagnostic clue in the clinical history is the speed with which the blood clears from the conjunctiva.

\section{References}

1. Conrads H, Kuhnhardt G. Zur Pathogenese der Lymphangiectasia Haemorrhagica Conjunctivae. Klin Monatsbl Augenheilkd 1957;131:670-4.

2. Leffertstra LJ. Lymphangiectasia hemorrhagica conjunctivae. Ophthalmologica 1962;143:133-6.

3. Stepanik J. Periodisch im Schlaf auftretende spontane Blutfullung der Bindehautlymphraume als Begleitsymptom eines Lymphhamangioms der Parotis. Klin Monatsbl Augenheilkd 1958;132:99-103.

4. Sugar HS, RA, Schaffner R. The bulbar conjunctival lymphatics and their clinical significance. Trans Am Acad Ophthalmol 1957;61:212-23. 